\title{
Is theory of yin-yang of traditional chinese medicine either monism or dualism?
}

\begin{abstract}
Philosophy is the study of general and fundamental problems concerning matters $\square$ The theory of Yin-Yang is the core issue of the theoretical system of Traditional Chinese Medicine (TCM). Qi is carrier of Yin and Yang, Yin and Yang is application of Qi. It reveals the law of changes in the universe and the law of nature that gives birth to life. Through the theory of Yin-Yang TCM practitioners understand and explain the origin and nature of life, the body's physiological function and pathological changes, guide the diagnosis and prevention of disease. Through reviewing the literatures of yin and yang theory, this article makes clear that the Yin-Yang theory of TCM is monistic rather than dualistic, its significance is to emphasize Yin and Yang mutual complementarity interaction unity of absolute and relative, interaction of partial and overall, complementarity of static and dynamic, couple hardness with softness, relevance of space and time, and to avoid absolute, one-sided or rigidification.
\end{abstract}

Keywords: theory of Yin-Yang of TCM, Qi, monism, dualism
Volume II Issue I - 2018

\section{Ben Bao Qi Cao}

Chair of the Board of College of Traditional Chinese Medicine Practitioners and Acupuncturists of British, Columbia

Correspondence: Ben Bao Qi Cao, Chair of the Board of College of Traditional Chinese Medicine Practitioners and Acupuncturists of British Columbia,Tel I604 6886 578, Email bbqc65@hotmail.com

Received: February 10, 2018 | Published: February 28, 2018

\section{Opinion}

Philosophy is the study of general and fundamental problems concerning matters such as existence, knowledge, values, reason, mind, and language. ${ }^{1}$ Zhou Yi Xi Ci (The Zhou Book of Change -The Survey)said: "Alternation between Yin and Yang is called Tao (Way)", ${ }^{2}$ Tao is the law of happen and development and changes for the things, "Yin-Yang 's greatness and vastness match the images of heaven and earth, change and transformation alternative four seasons, the meanings of the Yin-Yang movement and change of the sun and the moon, easiness and simplicity of the perfect philosophy in The Zhou Book of Change the perfect virtue; ${ }^{2} \mathrm{Li}$ Ji Li Yun (The Book of Rites The Conveyance of Rites)said: "From all this it follows that rules of ceremony must be traced to their origin in the Grand Unity. This separated and became heaven and earth. It revolved and became the dual force of Yin and a Yang (in nature). It changed and became the four seasons. ${ }^{3}$ Zhuang Zi $\bullet$ Za Pian Tian Xia (Zhuang Zhou Miscellaneous Chapters Tianxi) said: Yi (Book of Changes) is to show the action of the Yin and Yang;. ${ }^{4}$ Lao Tzu'sTao Te Ching ${ }^{5}$ said: "Tao gave birth to the One; the One gave birth successively to two things, three things, up to ten thousand. These ten thousand creatures cannot turn their back to the shade without having the sun on their bellies, and it is on this blending of the breaths that their harmony depends. ${ }^{6}$ The theory of Yin-Yang is the essence of ancient Chinese philosophy, and also the philosophical foundation of epistemology and methodology of TCM. It is the core issue of the theoretical system of $\mathrm{TCM}^{7}$ Through the theory of Yin-Yang TCM practitioners understand and explain the origin and nature of life, the body's physiological function and pathological changes, guide the diagnosis and prevention of disease. As the Huang Di Nei Jing. Su Wen (Yellow Emperor's Canon of Medicine Plain Conversation) said: "Yin and Yang serve as Dao (law) of the heavens and the earth, the fundamental principle of all things, the parents of change, the beginning of life and death and the storehouse of Shenming ${ }^{8}$ Alternatively it means that the law of Yin and Yang is the natural order of the universe, the foundation of all things, mother of all changes, the root of life and death. The theory of Yin-Yang is not only a simple philosophical theory, but also reveals the law of changes in the universe and the law of nature that gives birth to life. It is enough to see how important the theory of Yin-Yang is Huang Di Nei Jing •ling Shu (Yellow Emperor's Canon of Medicine • Spiritual Pivot said: "Clearly in the Yin-Yang, such as confused solution, drunk awake. ${ }^{9}$ Jing Yue Quan Shu Yin Yang said: "Medical doctrine, although complex, and can be said in a word: YinYang only. ${ }^{10}$ TCM practice must insist that Yin-Yang as the primary differentiation of disease, as the general outline of the eight principle of diagnosis, as the principle of herbs combination, as the foundation of formula and manipulation of acupuncture, and as both Yin-Yang in equilibrium as essentials of therapy. Is theory of Yin-Yang of Traditional Chinese Medicine either monism or dualism? Although Wikipedia defines "Yin and Yang as a dualistic concept derived from ancient Chinese philosophical thought, ${ }^{11}$ it also points out "The YinYang symbol in actuality has very little to do with Western dualism; instead it represents the philosophy of balance, where two opposites co-exist in harmony and are able to transmute into each other", "The complementary dualistic concept seen in Yin-Yang represent the reciprocal interaction throughout nature and exchange reciprocally to promote stabilization similar to homeostasis. ${ }^{11}$ Zheng Meng • Can Liang Pian said: "Qi is a thing of two bodies", "Qi consists of Yin and Yang, the reciprocal interaction of them gave birth successively ten thousand up to infinity. ${ }^{12}$ That is to say the world is the original One for all things, one Qi consists both of Yin and Yang, Qi movement is the movement of Yin and Yang, Qi is carrier of Yin and Yang, Yin and Yang is $\square$ application of Qi. Heng Qu Yi Shuo Shuo Gua Tai He Pian said: "Yin and Yang mutual complementarity interaction. The Yin and Yang consist of the deficiency and excess, moving and still, gathered and dispersed, also pure and turbid, the original One..$^{13}$ 
According to the theory of Yin-Yang, Qi is the most basic element that constitutes the cosmic and everything in the world. It is the origin of the world and the material basis for the unification of the infinite diversity of all things in the world. And also it is the foundation of unity of the body and spirit. Yin-Yang is the inherent property of Qi, the interaction of Yin-Yang of Qi is the root cause of Qi self-movement and the fundamental reason for the change of all things. Yang transforms qi while Yin shapes into configuration; Form (physical) and Qi (intangible) and their mutual transformation is the basic form of the physical world exists and movement. The material world, including life, is a movement world in which the Qi transformation, the rising and falling as well as moving in and out of Yin-Yang Qi, is constantly taking place. The emergence and disappearance of things are just the transformation of the form of existence of Qi. The theory of Yin-Yang reveals the fundamental laws of movement that exist in the two sides of things or things, which are in mutual compare at two poles, coexist and interdependence, waning and waxing, intercourse and inter-transformation, balance and harmonize. According to the definition of Monism in Wikipedia: "Monism is the philosophy that one origin of World is considered as a branch of ontology, ${ }^{14}$ we can define the theory of Yin-Yang as a monism. The significance to clarify that the theory of Yin-Yang in TCM is a theory of monism rather than dualism is to emphasize Yin and Yang mutual complementarity interaction, unity of absolute and relative, connection of partial and overall, complementarity of static and dynamic, couple hardness with softness, relevance of space and time, in order to avoid absolute, onesided or rigidification.

\section{Acknowledgment}

None.

\section{Conflict of interest}

The authors declare that there is no conflict of interest.

\section{References}

1. https://en.wikipedia.org/wiki/Philosophy

2. Shangwen Z, Huisheng F. The Zhou Book of Change II, P377-379. Hunan People's Publishing House. China; 2008.

3. https://ctext.org/liji/li-yun

4. https://ctext.org/zhuangzi/tian-xia

5. https://en.wikipedia.org/wiki/Tao_Te_Ching

6. Chen guying. Laozi, P87. Hunan People's Publishing House. China; 1999.

7. Luo Yuan-kai. Theory of Yin-Yang of Five Dynasties: The First Research Material of TCM Theory. People's Health Publishing House. Beijing: 1957.

8. Liu Xiru, Li Zhaoguo. Yellow Emperor's Canon of Medicine Plain Conversation, P57. Hunan People's Publishing House. China; 2005.

9. Liu Xiru, Li Zhaoguo. Yellow Emperor's Canon of Medicine Spiritual Pivot I, P544. Hunan People's Publishing House. China; 2008.

10. http://yibian.hopto.org/shu/?sid=67710

11. https://en.wikipedia.org/wiki/Yin_and_yang

12. http://club.ntu.edu.tw/ davidhsu/New-Davidhome/

13. https://zh.wikisource.org/zh-hant/

14. https://en.wikipedia.org/wiki/Monism 